\title{
EDUCATIONAL AND TRAINING EXPERIENCES IN GEOMATICS: TAILORED APPROACHES FOR DIFFERENT AUDIENCE
}

\author{
G. Tucci*, E. I. Parisi, A. Conti, M. Corongiu, L. Fiorini, F. Panighini
}

DICEA - Dept. of Civil and Environmental Engineering, University of Florence, Via di S. Marta 3, 50139 - Florence, Italy (grazia.tucci@unifi.it, ericaisabella.parisi@unifi.it, arch.a.conti@gmail.com, manuela.corongiu@unifi.it, lidia.fiorini@gmail.com, francesca.panighini@gmail.com)

\section{Commission V, WG V/1}

KEY WORDS: Geomatics, Photogrammetry, Cultural Heritage, Education, Training, Courses, Crowdsourcing, Learning-by-doing

\begin{abstract}
:
The recent outbreak of geospatial information to a wider audience, represents an inexorable flow made possible by the technological and scientific advances that cannot be opposed. The democratization of Geomatics technologies requires training opportunities with different level of complexity specifically tailored on the target audience and on the final purpose of the digitization process. In this frame, education plays a role of paramount importance, to create in the final users the awareness of the potentials of Geomatics-based technologies and of the quality control over the entire process.

This paper outlines the current educational offer concerning the Geomatics Academic discipline in the Italian higher education system, highlighting the lack of dedicated path entirely devoted to the creation of specifically trained figure in this field. The comparison with the International panorama further stresses out this necessity. The purpose of this work is to present different educational approaches by distinguishing between the starting knowledge level of the students/participants and the final aim of the training activities. Three main audiences have been identified: i) experts, who already know some basics of Geomatics to understand the theoretical concepts behind its technologies; ii) intermediate audience, who are interested in learning about Geomatics technologies and methodologies, without any previous or poor education concerning these topics; iii) non-experts, a mix of a wide group of people, with different educations and interests, or without any interest at all.

For each group, the multi-year experience concerning educational and training activities for the geomatics-based knowledge transfer in all the multi-level approaches of the GECO Lab (University of Florence) is presented.
\end{abstract}

\section{INTRODUCTION}

The Italian higher education system is organized in disciplinary groups and each University Academic belongs to a specific scientific sector. These groups are structured into levels in accordance with the Law 240/2010 (art. 15), listed from the most general to the specific ones: i) areas (14) ii) group of academic recruitment fields (88), iii) academic recruitment fields (188), iv) academic disciplines (367) ${ }^{1}$.

Among the current disciplinary sectors established by the Ministerial Decree n. 855/2015, the academic discipline ICAR/06 - Surveying and Mapping, is framed as reported in the attachment D of the Decree (Table 1$)^{2}$.

\begin{tabular}{|l|l|}
\hline Area & 08 \\
CIVIL ENGINEERING AND ARCHITECTURE \\
\hline $\begin{array}{l}\text { Group of academic } \\
\text { recruitment field }\end{array}$ & 08/A \\
LANDSCAPE AND INFRASTRUCTURAL \\
\hline $\begin{array}{l}\text { ENademic } \\
\text { recruitment field }\end{array}$ & 08/A4 \\
\hline GEOMATICS \\
\hline Academic discipline & ICAR/06 \\
& SURVEYING AND MAPPING \\
\hline
\end{tabular}

Table 1. Structure of the different levels which the academic discipline ICAR/06 belongs to, from the widest field (area) to the most specific one (academic discipline)
The thematic contents of this scientific field are related to georeferencing, geodesy, surveying and control, processing and restitution of complexes of metric and thematic data referred in space and time. The techniques included are aerial and groundbased photogrammetry, topography, photointerpretation of images, sequences, maps, remote sensing, GIS, space, aerial, sea and land navigation. All these topics have intrinsic multi-sectors applications and relate to different fields in terms of both interests and research ${ }^{3}$. The specific interests of Geomatics, in fact, refer to the acquisition, processing, restitution, analysis and management of metric or thematic data of earth surface or part of it, including also urban environment, infrastructures and built heritage. The application fields have, as subject, global and local reference systems, instruments and methods of survey, control and monitoring of the environment, structures and cultural heritage, treatment of measures, the production and updating of cartography and of topographic databases, mobile surveying systems, numerical models of terrain and surfaces, management and sharing of multi-temporal and multi-dimensional geographic information ${ }^{4}$.

\footnotetext{
* Corresponding author

${ }^{1}$ www.miur.gov.it

${ }^{2}$ Ministerial Decree n. 855/2015 - Attachment D

${ }^{3}$ Ministerial Decree $4^{\text {th }}$ October 2000 - Attachment B

${ }^{4}$ Ministerial Decree n. 855/2015 Attachment B
} 


\subsection{Geomatics courses in the Italian educational system}

The diffusion and distribution of ICAR/06 courses amongst Italian Universities are reported in Table 2, according to the Academic Association of Topography and Cartography (AUTEC) census of 2016.

The data clearly reports a higher diffusion of Geomatics courses in the biggest Italian Universities, as Polytechnic of Milan (49) and of Turin (27), followed by the Universities of Genoa (19) and Naples (17). A group of medium and small Universities follows in the list, with a number of courses ranging from 12 to 1 course (as an example, the University of Florence has 7 courses). This census shows how the distribution of Geomatics teachings is still strictly confined to singular courses among 139 different bachelors' (BSc) or masters' (MSc) degrees, from Architecture or Agricultural Science and Technology to Environmental Engineering or Archaeology.

\begin{tabular}{|c|c|}
\hline University & $\begin{array}{c}\text { ICAR/06 } \\
\text { n. courses* }\end{array}$ \\
\hline Polytechnic University of Milan & 49 \\
\hline Polytechnic University of Turin & 27 \\
\hline University of Genoa & 19 \\
\hline Parthenope University of Naples & 17 \\
\hline University of Padua & 16 \\
\hline University of Bologna & 15 \\
\hline Sapienza University of Rome & 14 \\
\hline University of Udine & 12 \\
\hline University of Turin & 10 \\
\hline University of Catania & 8 \\
\hline University of Palermo & 8 \\
\hline University of Florence & 7 \\
\hline Polytechnic University of Bari & 7 \\
\hline University of Pavia & 6 \\
\hline University of Parma & 5 \\
\hline University of Trento & 5 \\
\hline IUAV University of Venice & 5 \\
\hline University of Molise & 4 \\
\hline University of Bergamo & 4 \\
\hline University of Ferrara & 4 \\
\hline Marche Polytechnic University & 4 \\
\hline University of Modena and Reggio Emilia & 3 \\
\hline University of Perugia & 3 \\
\hline University of Pisa & 3 \\
\hline University of L'Aquila & 2 \\
\hline University of Cassino and Southern Lazio & 2 \\
\hline $\begin{array}{l}\text { University of Modena and Reggio Emilia / } \\
\text { University of San Marino }\end{array}$ & 2 \\
\hline University of Sassari & 2 \\
\hline University of Trieste & 2 \\
\hline University eCampus & 2 \\
\hline Mediterranea University of Reggio Calabria & 2 \\
\hline University G. D'Annunzio - Chieti-Pescara & 1 \\
\hline University of Lecce & 1 \\
\hline Carlo Bo University of Urbino & 1 \\
\hline Niccolò Cusano University & 1 \\
\hline
\end{tabular}

Table 2. List of Italian Universities and the respective number of courses in the academic discipline ICAR/06.

*The data reported refers to the year 2016

Keeping the track of the evolution of the didactic system within Italian Universities requires a periodic census, similar to the one reported above. A new census is under elaboration by AUTEC and should be released in the current year.

The main aspect evidenced by the AUTEC census is that the presence of geomatic disciplines is only related to singular courses amongst already existing BSc or MSc degrees. A dedicated educational path devoted to the creation of a specifically trained figure in this field does not exist it Italy, yet. In-depth paths related to geomatic discipline are usually demanded to post-academic programs.

Furthermore, the formative lack and the scarce space reserved to geomatic topics in the study plans of future architects and engineers, results in ambiguities of language and absence of shared protocols between different sectors which use the same data. On the professional side, the forced need for chasing the technological evolutions prevent them to stay constantly updated in terms of both tools and knowledge.

\subsection{The necessity of a dedicated path}

The rapid evolution in the recent decades of Geomatics technologies, products and services requires that the educational system properly follows this growing increase of at least $35 \%$ per year on the global market ${ }^{5}$. The growth estimations for the overall Geospatial Market go from $\$ 300$ billion in 2017 to $\$ 500$ billion by 2020, at a Compound Annual Growth Rate (CAGR) between $15-20 \%$ (GeoBuiz 2018). This noticeable growth is driven by technological improvements, democratization of geospatial information and integration with advancing digital technologies, as Big Data, Cloud, Artificial Intelligence, Internet of Things, Augmented Reality and Virtual Reality and Automation. In this context, new professionals and reference figures are needed to face the fast evolution of market trends, stay ahead of competition and fill the gap between demand of and supply of professional education in terms of both quantity and quality (Masum et al. 2017)

The role of Geomatics and surveyors has been changing since centuries, to adapt to the technological evolutions and to face the constant challenges of the "Science of Where". The traditional role of a surveyor able to collect, analyze, visualize and interpret spatial data with the most up-to-date instruments and software has to be improved and supported by further skills. The final aim is to train highly specialized, reliable and conscious professional figures able to manage and understand the rigorous processes behind the automatisms, to control and modify them by providing information technologies skills, not simple operators unaware of the contents trained to press the buttons of software to produce 3D models. The intellectual integrity, the documentation of the processes, the sustainability and accessibility of the produced outputs, the metadata, are all conceptual aspects that can not be divided from the metric ones (Tucci 2016).

The educational process should also empower the trained professionals in the decision-making process, by properly considering the enormous quantity of collected georeferenced data. These figures will have to play a key role also in the environmental, engineering, architectural management and administration with the same level of consideration of other disciplines.

Another fundamental aspect that should characterize a complete figure educated in the Geomatics field should be the ability to adapt in transdisciplinary contexts, which is proper of Geomatics world. In the current panorama of a liquid world, where each discipline is subjected to hybridization and osmosis of contents depending on their applications, the impellent need is a figure which reflects the multidisciplinarity of the training process also on the final interaction with other sectors and professionals (Tucci 2017). It is also essential to find a shared language with the other sectors' operators to define mutual necessities and find shared solutions to overcome boundaries, thus easing the transdisciplinary communication, since Geomatics services often represent the only connection between analytical, management and planning activities.

\footnotetext{
${ }^{5}$ www.marketsandmarkets.com
} 


\subsection{Comparison with the International panorama}

The comparison of Geomatics dedicated path in University education between Italy and the International panorama can be clearly summarized in Figure 1. The dots refer to MSc (Master of Science) programs distributed all over the world (Figure 1 a) and in Europe (Figure $1 \mathrm{~b}$ ). At the present situation, Italy does not have any specific MSc program completely devoted to the training and education of a Geomatics professional or research figure (data updated to 2017).

As reported by the Geospatial Industry Outlook \& Readiness Index of 2018, "a geo-intelligent workforce and higher education institutional capacity is a key determinant of a country's geospatial domain strengths". The Institutional Capacity of 50 selected countries was evaluated by considering:

a) Geospatial Science and Technology courses at Post Graduate and Research (Ph.D.) levels

b) Geospatial Domain Graduation, Diploma (1-3 years) and Certificate courses (graduate and postgraduate levels)

The comparison process has been carried out by considering the educational offer in geospatial domain science and research as well as application-oriented courses. The results of the report highlight more virtuous Countries (Leaders), as US, UK, Germany, The Netherlands, where the education offers a wide range of doctorate, post-graduate and graduate programs in the field of geospatial education as pure and interdisciplinary courses. As an example, amongst European Countries, the Netherlands have 5 Masters of Science completely dedicated to Geomatics for Built Environment (Delft University of Technology), Geoinformation Science (Wageningen University), Geoinformation and Earth observation (University of Twente), Geographical Information Management and Applications (Utrecht University), Geographical Information Science (Vrije Universiteit Amsterdam). The other Countries are listed as Challengers and Aspirers. Italy's position rank is in the middle (24), among Challengers, after France and Malaysia. As previously stated, although the educational offer in the Geospatial Domain has constantly being improved, it is still relegated to singular and isolated courses within already existing Schools, as Engineering, Architecture, Sciences, and Information Technology Schools.

In addition to University Education, also International organizations, such as the International Society for Photogrammetry and Remote Sensing (ISPRS) has a role in the spreading of education concerning Geomatics ${ }^{6}$. The Technical Commission V (TC V) is specifically devoted to this mission, i.e. to support, promote and motivate Capacity building in Geoinformatics education and training, at different levels of professionals, educators and students and develop cost-effective teaching methods which include distance learning programmes, International collaborative effort for sharing web-based resources - both material and trainers as well as best practices for effective transfer of knowledge and curricula development that meets regional needs, encourage open source software tools and innovative methodologies for urban infrastructure, citizen science and geospatial technology in various applications, and support youth forum and students consortium activities (Gong et al. 2017).

Other Organizations, as International Federation of Surveyors ${ }^{7}$ (FIG) - Commission 2, Accreditation Board for Engineering and Technology ${ }^{8}$ (ABET) for the US and the Royal Institution of

\footnotetext{
${ }^{6}$ www.isprs.org

${ }^{7}$ www.fig.net

${ }^{8}$ www.abet.org
}

Chartered Surveyors (RICS) ${ }^{9}$ for UK, are devoted to educational activities and capacity building, for accreditation of standards in university programs and the suitability of their offer to sustain the professional needs, respectively (Masum et al. 2017).

(a)

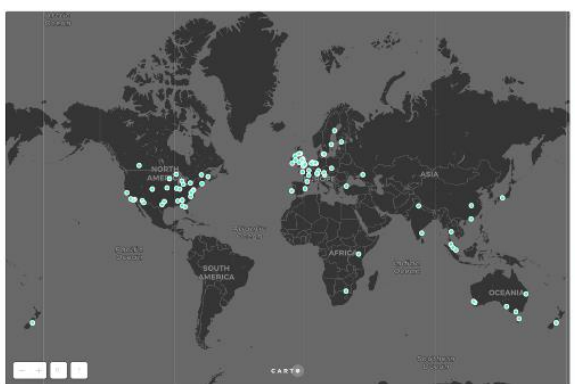

(b)

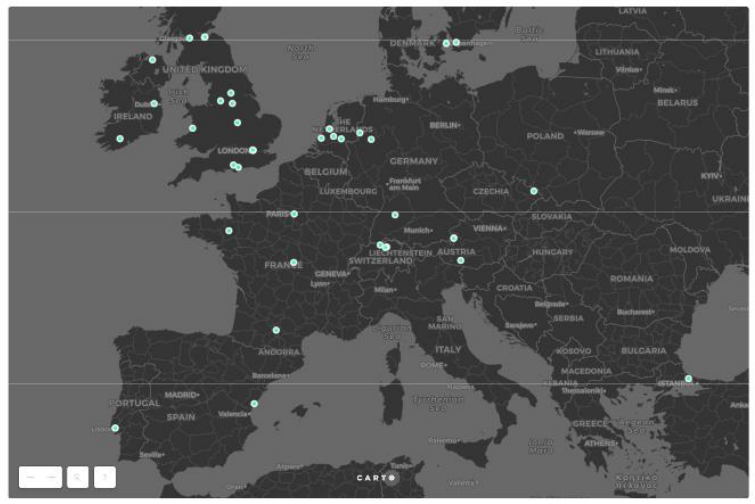

Figure 1. a) The data showed in the map ${ }^{10}$ show the distribution of Geomatics BSc and MSc in the world. b) The same distribution $n$ Europe: the absence of specific programmes in Italy is evident.

\section{EDUCATIONAL OFFERS}

The current educational offer concerning Geomatics techniques in Italy ranges from high level of knowledge transfer into academic Institutions to professional or short courses. A distinction between different educational approaches has to be made by considering the starting knowledge level of the students/participants and the final aim of the training activities. Three main didactic approaches can be identified by considering the level of knowledge of specific topics related to Geomatics:

an expert audience is supposed to already know some basic Geomatics concepts or, at least, the methodological basis to understand the theoretical concepts behind its technologies. This audience usually comes from academic education, as Engineering, Architecture, Sciences, and Information Technology Schools, where one or more courses related to these topics should be present. Moreover, an expert audience can be made of professionals, with several years of experience on the field and a specific interest in deepening and updating their knowledge to keep up with the market evolution;

an intermediate audience, instead, might be interested in learning about Geomatics technologies and methodologies, without any previous or poor education concerning these topics. The interest could be only in having an overview of what Geomatics is, its potentialities and applications. On

\footnotetext{
${ }^{9}$ www.rics.org

${ }^{10}$ www.geoawesomeness.com
} 
the other hand, the interest could be very focused on a specific topic within the several Geomatics fields (i.e. remote sensing instead of topography, ground-based vs aerial surveying techniques, etc.). The provenience of this kind of audience can be both from the Academia (students) and the Professional worlds;

a non-expert audience could be a mix of a wide group of people, with different educations and interests, or without any interest at all. In this case the purpose is both to let them know the existence of such technologies and to interest them in further deepen their knowledge about Geomatics. A target audience can be chosen to direct a specific activity, by using their background to link them with Geomatics topics. As an example, amateur or professional photographers, high school students, video-makers, association for heritage protection, citizens, and so on can be endeared and involved in collaborative approaches.

\subsection{Expert audience}

The current Italian University System (D.M. 509/99; D.M 270/04) is organized as showed in Figure 2. The University courses are divided into three-years programme (BSc) + twoyears (MSc) degrees. Each level requires a specific number of university formative credits (CFU) to be overcome: $180 \mathrm{CFU}$ for the first level degree $+120 \mathrm{CFU}$ for the second level, for a total of $300 \mathrm{CFU}$.

The Bachelor of Science degree's purpose is to give the theoretical and practical basis of a specific field, to enter the professional job market or to continue the studies, by applying to the Master of Science programme, to a $1^{\text {st }}$ level university master's degree or to Post-graduate programme. The Master of Science degree, instead, aims to deepen the knowledge and provide rigorous and advanced training in specific sectors. After obtaining the MSc, it is possible to apply for a $\mathrm{PhD}$ programme (3 years), $2^{\text {nd }}$ level Master or Post-graduate programme ${ }^{11}$, as reported in Figure 2 (Achille et al. 2018).

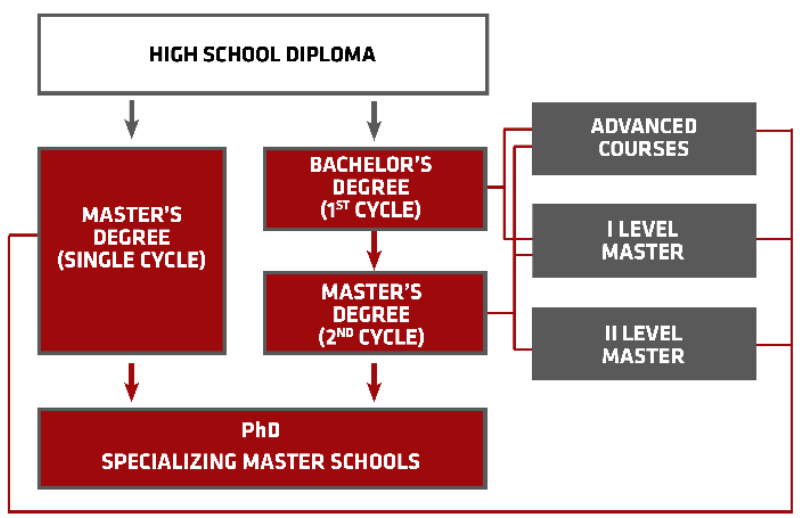

Figure 2. The scheme represents the Italian University system, reporting also post-academic programmes

Post-graduated programmes usually include Specializing Masters schools (Scuole di Specializzazione) and Continuing Education Programmes, as Advanced courses (Corsi di Perfezionamento). These courses aim to deepen the knowledge and skills required for particular professional activities, during at least 2 years. They qualify people to work in such professions and train specialists for certain professional sectors.
Specific training programmes for an expert audience, are represented by:

\author{
University educational offer \\ Post-graduated Master courses (first- and second-level) \\ Specializing Masters schools \\ Advanced courses
}

This high-level and long-term education provides complete theoretical basis on Geomatics techniques (i.e. topography, laser scanning, photogrammetry, mobile mapping, GNSS, etc.), to give the students an adequate knowledge of the methodologies and a suitable training on the field. The most common didactic approach for this kind of courses, is a combination of in-class ex cathedra lectures with practical exercises. (Cuca 2018).

\subsection{Intermediate audience}

Short-term courses for an audience with basic knowledge in the Geomatics topics can be provided in the form of:

$$
\begin{array}{ll}
\text { - } & \text { Seminars } \\
\text { - } & \text { Tutorials } \\
\text { - } & \text { Workshops }
\end{array}
$$

In this case, the level of detail of knowledge transfer will be proportional to the duration of the educational course: a twoweeks workshop may be enough to properly train the participants in the use of specific techniques, while a three-day tutorial may give only an overview on some topics. The lack of time available usually needs a different and more effective approach from the traditional in-class ex cathedra lessons, mainly focused on the "learning-by-doing" method (Tucci et al. 2018a).

The participants are, in this way, involved in the training activities by doing practical experiences on the field, possibly focusing on a case study related to their heritage, thus reinforcing their engagement (Kravchenko et al. 2016). Practice is supported by short lectures on the basis behind the applied methodologies and the used technologies. The participants receive, also, suitable didactic material to further sustain the knowledge and know-how transfer process. This approach has proved to be effective as learning and teaching strategy since it triggers the problemsolving skills of the students, who are forced to find suitable solution for real problems, which will help the development of their decision-making and critical analysis skills (Kosmatin et al. 2016).

\subsection{Non-expert audience}

Crowd-sourced approaches are addressed to completely nonexpert audiences, interested in learning Geomatics techniques. In this case, no basic knowledge is required and the participants are trained during social and collaborative events on a specific part of the workflow, e.g. imagery acquisition of the photogrammetry process. The final aim is to make the participants aware of the existence of these techniques, which can be further deepened or used for creative purposes, with considerable benefits also under the social aspect, such as community engagement, social interaction and active involvement.

The current digital democratization flow is a trend that cannot be opposed although a "culture of positioning" is not as much diffused. One effect is that more than 2.8 billion of smartphones are predicted to circulate all over the world by 2020 , each with advanced positioning capabilities and increasing camera resolutions. This would provide locationing tools and photogrammetric potential devices in everyone's hands, but who will really be able to evaluate the quality and the accuracy of the results?

\footnotetext{
${ }^{11}$ www.esteri.it
} 
Furthermore, the European Digital Single Market ${ }^{12}$ aims at boosting the use of digitization capacities of the Member Countries to improve economy, diffuse culture and foster the sharing of the outputs with the community, removing geoblocking and copyright issues (i.e. through Europeana ${ }^{13}$ ). Also, academic initiatives use a crowdsourced approach to digitize museum collections, usually not all visible, to create a digital repository for making them available to both researches and community (Guidi et al. 2018).

This democratization process has ambivalent effects, since it can be positively used for collaborative approaches thus generating a virtuous circle of social wellness because it empowers people's awareness of their culture by triggering collective consciousness, identification and engagement feelings (Chiesi et al. 2015, Latellier 2015); on the other hand the risk of lack of quality and awareness of the metric control over the resulting outputs is evident.

For these reasons the spreading of controlled training activities also to non-experts can make people aware of the existence of such techniques, thus triggering their potential interest in Geomatics technologies. To prevent the uncontrolled introduction on the market of 3D models, the role of education becomes of paramount importance, thanks to the supervision by experts and specialists, who provide tools, technology, and training by the diffusion of best practices, shared standards and suitable guidelines to a broad audience, thus helping a sort of quality control (Bitelli et al. 2017).

\section{THE GECO LAB EXPERIENCE}

Since 2008, the Geomatics for Environment and Conservation of Cultural Heritage (GECO) Laboratory belonging to the Department of Civil and Environmental Engineering of the University of Florence is active in the field of digital surveys of Cultural Heritage for documentation, conservation and management purposes. It also has gained a multi-year experience concerning educational and training activities for the geomaticsbased knowledge transfer in all the multi-level approaches listed above. An experience of the GECO Lab for each approach will be presented below.

\subsection{Educational experiences for expert audience}

University courses in the Academic discipline ICAR/06 are annually held both in the Architecture and Engineering Schools of the University of Florence. A progressive increase can be observed, from the 7 courses of 2016 to a total of 8 courses currently (academic year 2018-2019) being teached and a further increase to 9 for the next year.

The courses held at the Architecture School are mainly configured as interdisciplinary blended laboratories (e.g. as part of restoration laboratories). Since these courses are mainly characterized by a strong applicative character it is more difficult to give the theoretical details of the discipline. On the other hand, the students have to work in group to produce a final project, which is a more useful approach for their future professional needs as restorers and architects.

In the Engineering School there are few spaces left in the institutional curricula for Geomatics discipline, where the courses are increasingly reduced. But in this context, the students have a stronger background to learn the theoretical basis to understand also what is behind the processes. The topics and the teaching approach also vary by considering the specific curricula of the Civil, Building and Environmental Engineering courses. As an example, the courses held in the Environmental curriculum will be more focused on aerial photogrammetry, remote sensing and GIS compared to the topics more suitable for civil or building engineering (i.e. laser scanner, close-range ground-based photogrammetry, etc.).

A first level master (see Figure 2) "Production and processing of ground- and UAV-based 3D data" of one year was held in 2017, bringing together different backgrounds coming from engineering, architecture, archaeology, geology, agronomy and cultural heritage fields. The aim of this post-graduate programme was to enhance the specific skills acquired in the previous educational path, by integrating them with digitization project planning and management skills of the built heritage and the environment with both ground-based and aerial (UAV) technologies. The programme of the course had been structured by particularly focusing on the most innovative Geomatics methodologies and technologies, such as: i) multi-sensor acquisition of spatial data, ii) UAV photogrammetry and photointerpretation, iii) GIS and data classification, iv) positioning systems, v) cartography for the representation and management of environmental data, vi) build heritage and environmental modelling. A final traineeship of 125 hours concluded the first level Master course with a final dissertation on the application of the methodologies learned.

Moreover, Advanced traineeships courses have been held for five years (academic years 2009-2010, 2011-2012, 2012-2013, 2013 $2014,2014-2015$ ), with the specific aim of deepen the knowledge of technologies and methodologies developed in the Geomatics research. The courses were organized in a theoretical frame and significative practical activities, open to graduates and professionals to give them the necessary knowledge and skills to deal with the topic of the 3D measure, meaning it as a critical choice and the result of an interpretative process of the phenomenon to be modelled, as an essential basis for any intervention on built heritage or environment. The duration of these courses was usually limited to a couple of months and to about 10-12 participants, with a final applicative workshop to apply to practice their learning (Figure 3 ).

Finally, a course has been held also in the Specializing Master School in Archaeology (which lasts two years), Topography and Cartography - Remote Sensing for the environmental archaeology (10 hours) to provide the archaeologists the theoretical basis and skills in the concept of spatial measures with integrated surveying technologies.

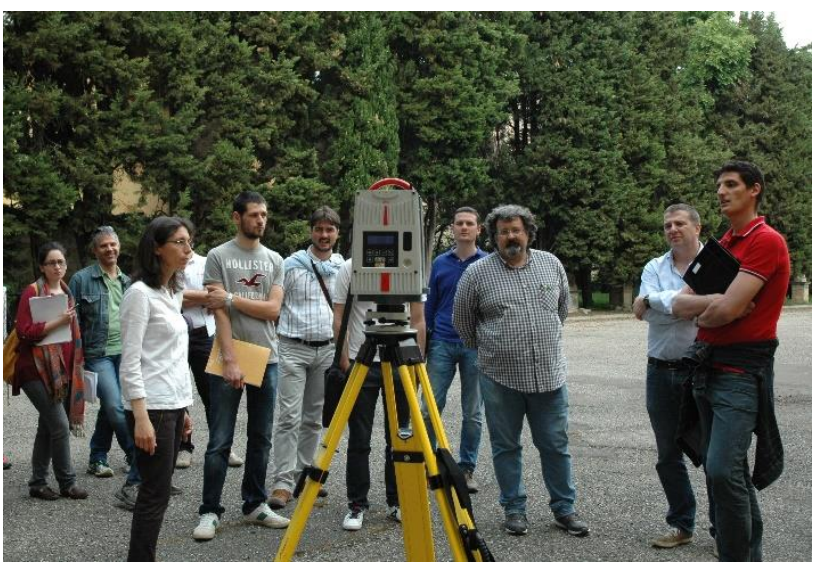

Figure 3. Some activities on the field with a laser scanner during the Advanced Course of the academic year 2014-2015. 


\subsection{Educational experiences for intermediate audience}

For what concern the learning-by-doing approach two international educational experiences held in 2018 will be presented as examples of this approach: i) a two-weeks workshop within the INNOVA CUBA project in Havana (Cuba) and ii) a three-days tutorial during the ISPRS TCV Mid Term Symposium in Dehradun (India).

The two experiences were quite different, since the final purpose of the "INNOVA CUBA - International and intersectoral intervention for the safeguard of the cultural heritage of the country" project is the promotion and use of technological and methodological innovations in the conservation and protection of cultural heritage, to strengthen the touristic offer and the economical and social growth of the Cuban territory ${ }^{14}$ (Tucci et al. 2018b).
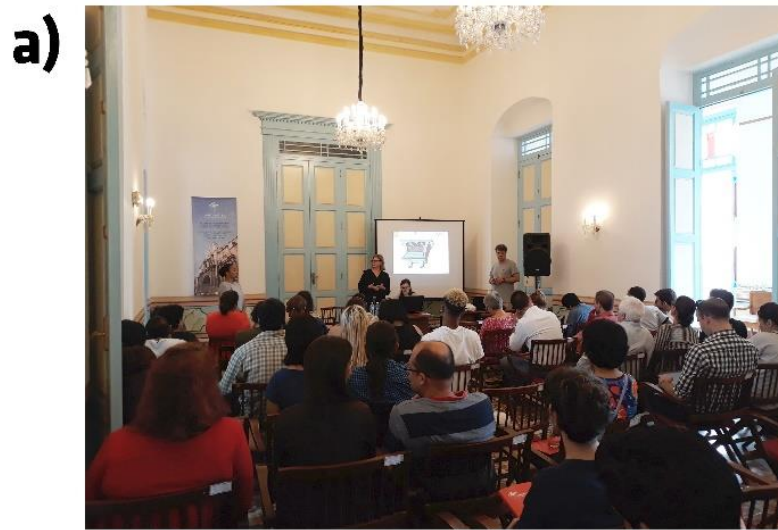

b)

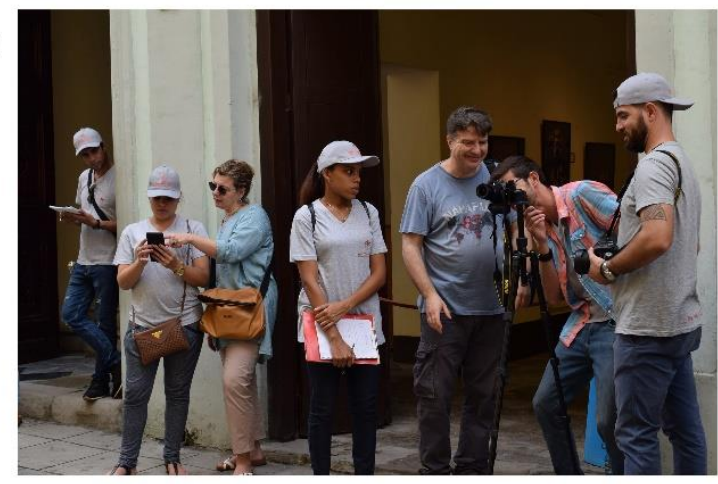

Figure 4. a) The final seminar in Havana after the two-weeks course. b) Surveying activities on the field by using the "learning-by-doing" approach

The educational activities were carried out during the mission of a research group of GECO Lab in Cuba, from January $20^{\text {th }}$ to February $4^{\text {th }}, 2018$. The two-weeks tutorial has involved twelve participants, belonging to different backgrounds in the field of engineering, architecture, anthropology, photography, and cartography. The principal goal has been to give the participants

14 The project "INNOVA CUBA - International and intersectoral intervention for the safeguard of the cultural heritage of the country" has started in July 2017. The project is funded by AICS - Italian Agency for Development Cooperation and coordinated by the ONG ARCS Culture Solidali. Amongst the partners: Oficina del Historiador de la Ciudad de La Habana $(\mathrm{OHcH})$, Assorestauro, the Academy of Fine Art of Varsavia, the University of Florence with DIDA - Department of Architecture and DICEA - Department of the cognitive instruments for being able to autonomously choose the suitable surveying techniques depending on i) the characteristics of the object and ii) the available instruments, iii) to correctly plan the acquisitions, iv) to evaluate the metric quality of the outputs. The two-weeks activities were organized in La Habana Vieja neighbourhood, by choosing cases of study to be surveyed with limited sizes, as portals, facades and small objects (Figure 4). The course was structured as in the following steps:

- Theoretical lectures

- Surveying activities on the field

- Data processing

- Final workshop

For what concern the other project, as reported by Gong et al. (Gong et al. 2017), in the last years several educational activities were carried out by the TC V, as i) web-based resource-sharing, ii) E-delivery of education services, iii) international collaborative education programs, iv) regional cooperation activities, v) profession development for young people, and vi) capacity development.

The research group of the GECO Lab has participated to the iii) activity as Coordinator of the Education and Capacity Building Initiatives 2018, with the ISPRS funded project "Education and Training Resources on Digital Photogrammetry"15. The project provides for preparing and sharing some didactic material, in particular about digital close-range and UAVs photogrammetry, aimed to cultural heritage documentation, structural and seismic performance assessment and support to conservation projects.

The "Ground based 3D Modeling (Close Range Photogrammetry $\&$ TLS)" tutorial was held during November $17^{\text {th }}-19^{\text {th }}$ and represented an opportunity for 15 participants, mainly with a remote sensing background, to deepen their knowledge and practice in the use of up-to-date reality-based 3D technologies: digital photogrammetry and Terrestrial Laser Scanner (TLS) to produce $3 \mathrm{D}$ models with metric quality (Figure 5 ). The tutorial activities were divided in:

- on-the-field data acquisition, for the digitization of a significant case of study placed in Dehradun, the Forest Research Institute, through both laser scanner and closerange photogrammetry techniques

in-class basic theoretical lectures about 3D surveys and ground based technologies; overview of data processing workflow and software.

For both these educational experiences, a final feedback was requested to the participants, in order to give the traineers information to further calibrate or adapt the educational approach. The results of the anonymous assessment questionnaire submitted to the twelve participants of the INNOVA CUBA experiences are reported in Tucci et al. (Tucci et al. 2018b), while the Indian participants to the tutorial were asked to give only oral feedbacks.

Civil and Environmental Engineering, AITR - Italian Association for Responsible Tourism and ARCI Toscana.

15 The "Education and training resources on digital photogrammetry" ISPRS project has been held together with Anjana Vyas (CEPT University), Vikram Sorathia (Kensemble Tech Labs), Satwant Rihal (California Polytechnic State University). The project aim is to produce and share new and updated educational and training resources useful for supporting tutorials, workshops, seminars about the latest advances in digital photogrammetry. 


\subsection{Educational experiences for non-expert audience}

The last approach has been recently tested within the INNOVA CUBA project in Havana, by involving a selected audience with no previous experience in close-range digital photogrammetry. On 27-28 June 2018 the Cuban partners organized a first test event of collaborative photogrammetry in Havana. The participants were 15 students of the fourth year of the CUJAE Universidad Tecnológica de La Habana. The event was organized in two days: the first day was dedicated to explaining theoretical basic concepts, such as elements of photography, basics of photogrammetric surveying, software tutorial and the theme of collaborative photogrammetry, by recalling the structure of the course. The second day was dedicated to pictures acquisition on the field and data processing; specifically prepared guidelines were distributed as a support for the images acquisition process around the city.

The workshop was entirely organized and teached by four participants to the first training, whom became trainers in turn. This first experience represented an applicative test of the two different aims of this project: on one side an in-depth training for specialized technicians who have to deal with conservation issues and will be future trainers in turn, on the other the transfer of basic concepts about photogrammetry (by focusing on the acquisition phase) to a wider audience in little time.

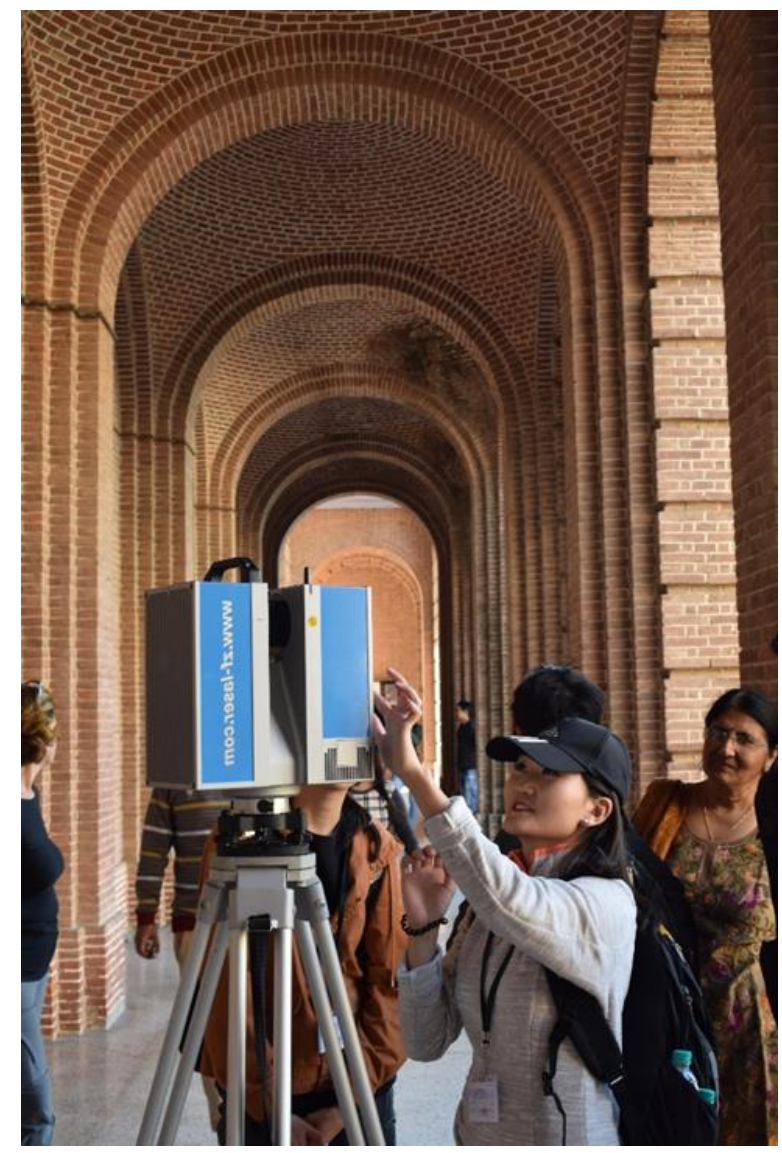

Figure 5. One participant of the "Ground based 3D Modeling (Close Range Photogrammetry \& TLS)" tutorial during the surveying activities at the Forest Research Institute of Dehradun

\section{CONCLUSIONS}

In conclusion, the previsions of future market evolutions expect a loss of the traditional identity of the Academic discipline and the redefinition of roles, directions and way of participation to the activities of digitalization already underway all over the world. New services and policies will have to substitute to the previous primary and secondary roles, driven by the democratization of technologies and the spread of open data, big data, crowdsourcing and social participation for the building of the digital heritage.

In this frame education plays a fundamental role, to update the traditional role of the surveyor from not only an expert operator to a highly specialized, reliable and conscious figure able to manage and understand the rigorous processes behind the automatisms. The current situation in the Academic Italian system has been also reported, highlighting the presence of geomatic disciplines only related to singular courses amongst already existing BSc or MSc degrees. Therefore, the necessity of a dedicated educational path devoted to the creation of a specifically trained figure in this field has been also underlined, also compared to the International panorama, where these courses are already a norm.

An in-depth analysis of the present Geomatics educational offer in Italy has been also given, ranging from high level of knowledge transfer into academic Institutions to professional or short courses. Different educational approach has been presented by considering the starting knowledge level of the participants and the final aim of the training activities, thus dividing the audience in experts, intermediate and non-experts. Three main didactic approaches have been identified for each one and the experience of the GECO Lab reported respectively with some practical examples.

Future perspectives should certainly continue the efforts in the educational strengthening and diffusion, aiming to the definition of a dedicated career for this discipline, by trying different approaches, as an example the institution of Professionalizing Courses for specific training of figure able to compete with the International panorama. In the meantime, the diffusion of Geomatic courses within already existent BSc or MSc degrees is a first step to consolidate the importance of this discipline.

Furthermore, increasing participating social events of crowdsourcing are going to be organized, both as the main output of the next mission within the INNOVA CUBA as well as in the \#3DHackathon project for high school students. The use of photogrammetry to digitize the local cultural heritage, by using specifically prepared guidelines and basics theoretical concepts, will be tested to train non-expert audiences.

\section{ACKNOWLEDGEMENTS}

This work is based upon work supported by the "GAMHer project: Geomatics Data Acquisition and Management for Landscape and Built Heritage in a European Perspective", (PRIN2015 n.2015HJLS7E).

\section{REFERENCES}

Achille, C., Fassi, F., Mandelli, A., Fiorillo, F., 2018. Surveying cultural heritage: summer school for conservation activities. Applied Geomatics, Special Issue GeoRes2017, 10(4), 579-592

Bitelli, G., Balletti, C., Brumana, R., Barazzetti, L., D’Urso, M. G., Rinaudo, F., Tucci, G., 2018. Metric Documentation of Cultural Heritage: research direction from the Italian GAMHER project. Int. Arch. Photogramm. Remote Sens. Spatial Inf. Sci., XLII-2/W5, 83-90. doi.org/10.5194/isprs-archives-XLII-2-W583-2017 
Chiesi, L., Costa, P., 2015. One strategy, many purposes. A classification for cultural mapping projects, Cultural Mapping as Cultural Inquiry, Routledge, London, 69-85

Cuca, B., 2018. Geo-Spatial Information and Geomatics applications in Higher Education: an overview of main trends and recent changes. Proc. SPIE 10773, Sixth International Conference on Remote Sensing and Geoinformation of the Environment (RSCy2018), 1077307 (6 August 2018). doi.org/10.5194/isprsarchives-XLI-B6-87-201

GeoBuiz - Geospatial Industry Outlook \& Readiness Index, 2018 Report of Geospatial Media + Communication

Gong, J., Yue, P., Woldai, T., Tsai, F., Vyas, A., et al., 2017. Geo-spatial Information Science Geoinformatics education and outreach: looking forward. Geo-spatial Information Science, $5020,1-9$

Guidi, G., Malik, U. S., Frischer, B., Barandoni, C., Paolucci, F., 2018. The Indiana University-Uffizi project: Metrologica! challenges and workflow for massive 3D digitization of sculptures. Proceedings of the $201723^{\text {rd }}$ International Conference on Virtual Systems and Multimedia, VSMM 2017, $1-8$

Kosmatin Fras, M., Grigillo, D., 2016. Implementation of active teaching methods and emerging topics in photogrammetry and remote sensing subjects. In: The International Archives of the Photogrammetry, Remote Sensing and Spatial Information Sciences, Vol. XLI-B6, 87-94

Kravchenko, I., Luhmann, T., Shults, R. 2016. Concept and Practice of Teaching Technical University Students to Modern Technologies of 3d Data Acquisition and Processing: a Case Study of Close-Range Photogrammetry and Terrestrial Laser Scanning. The International Archives of the Photogrammetry, Remote Sensing and Spatial Information Sciences, XLI-B6, 6569. doi.org/10.5194/isprsarchives-XLI-B6-65-20

Letellier, R., 2015. Recording, documentation and information management for the conservation of heritage places. Routledge, London/New York

Masum, F., Groenendijk, L., Mansberger, R., 2017. Enhancing the Role of Surveyors: Bridging the Gap between Demand for and Supply of Professional Education. Proceedings of FIG Working Week 2017, Surveying the world of tomorrow - From digitalisation to augmented reality Helsinki, Finland, May 29June 2, 2017

Tucci, G. 2016. Geomatics and Digital Heritage. From dataset to models: what next? SIFET Bulletin 3 (2016): Sezione Scienza

Tucci, G., 2017. GeoRes 2017: nuove sinergie tra Geomatica e Restauro. Special Issue GeoRes2017, Altralinea Edizioni, Firenze, ISBN: 978-88-94869-22-4

Tucci., G., Conti., A., Fiorini., L., Panighini., F., Parisi., E. I., 2018a. Education and training resources on digital photogrammetry. Int. Arch. Photogramm. Remote Sens. Spatial Inf. Sci., XLII-5, 45-50. doi.org/10.5194/isprs-archives-XLII-545-2018

Tucci, G., Conti, A., Fiorini, L., Mei, F., Parisi, E. I., 2018 b. Digital photogrammetry as a resource for Cuban Cultural Heritage: educational experiences and community engagement within the INNOVA CUBA project. Int. Arch. Photogramm. Remote Sens. Spatial Inf. Sci., XLII-5, 37-44. doi.org/10.5194/isprs-archives-XLII-5-37-2018

Revised March 2019 